\title{
Lumen
}

Selected Proceedings from the Canadian Society for Eighteenth-Century Studies

\section{La France foutue : allégorie « foutative " et rapport à l'histoire}

\section{Stéphanie Massé}

Volume 25, 2006

URI : https://id.erudit.org/iderudit/1012087ar

DOI : https://doi.org/10.7202/1012087ar

Aller au sommaire du numéro

Éditeur(s)

Canadian Society for Eighteenth-Century Studies / Société canadienne d'étude du dix-huitième siècle

ISSN

1209-3696 (imprimé)

1927-8284 (numérique)

Découvrir la revue

Citer cet article

Massé, S. (2006). La France foutue : allégorie « foutative » et rapport à l'histoire. Lumen, 25, 221-231. https://doi.org/10.7202/1012087ar

Copyright (c) Canadian Society for Eighteenth-Century Studies / Sociéte canadienne d'étude du dix-huitième siècle, 2006
Ce document est protégé par la loi sur le droit d'auteur. L'utilisation des services d'Érudit (y compris la reproduction) est assujettie à sa politique d'utilisation que vous pouvez consulter en ligne.

https://apropos.erudit.org/fr/usagers/politique-dutilisation/ 


\section{La France foutue : allégorie «foutative» et rapport à l'histoire}

Voici, en quelques mots, l'exposition du troisième et dernier acte de $\mathrm{La}$ France foutue, curieuse tragédie lubrique et royaliste vraisemblablement écrite à la toute fin du XVIII ${ }^{\mathrm{e}}$ siècle. Pendant que le comte de Puisaye, général des Chouans, "assis sur un canapé, veut y branler la Vendée qui s'en défend ${ }^{1}$ ", l'Angleterre, "de l'Ancienne Albion, illustre maquerelle ${ }^{2}$ », et tenant bordel chez le duc Philippe d'Orléans, invite Frédéric-Guillaume III roi de Prusse, François II empereur d'Allemagne et Charles IV roi d'Espagne à dépuceler la France qui, dans un long monologue tragique, s'exclame :

Je suis enfin foutue !...et ces triples couleurs,

Symbole de ma honte et de tous mes malheurs,

De cet affreux bordel, sont, hélas ! la livrée...

A quels tristes excès me vois-je enfin livrée!

Mon voile était sans tache, et seul il était blanc!

Son éclat précieux est souillé par le sang.

De vierge que j'étais me voilà république ;

Je suis à tout le monde et ma honte est publique.

1 Anonyme, La France foutue, tragédie lubrique et royaliste en trois actes et en vers, Paris, Les Belles Lettres, coll. «Le corps fabuleux», 1995 [ca. 1802], exposition du troisième acte, p. 74. Cette pièce anonyme dont la paternité fut accordée à Sade, selon Paul Lacroix, et à un certain abbé Proyard, à la faveur d'une note manuscrite figurant sur l'un des deux exemplaires de la Bibliothèque nationale de France, figure dans la Table des pièces de théâtre décrites dans le catalogue de la Bibliothèque de $M$. de Soleinne de Charles Brunet, New York, Burt Franklin, 1914, au numéro 3876. J'ai également pu consulter l'un des exemplaires, conservé à la réserve du site François-Mitterand de la Bibliothèque Nationale de France à la cote ENFER 652. Il s'agit d'un exemplaire unique de la France foutue où apparaît un exorde manuscrit au début de la pièce ; il aurait appartenu au comédien Talma.

2 Ibid., Acte I, sc. 1, p. 25. 
Chacun me fout, chacun veut être mon fouteur, Et personne ne craint d'alarmer ma pudeur.

Sans égards, oubliant mes droits de souveraine,

Sans états et sans nom, l'on me traite en romaine ${ }^{3}$.

Que penser de cette singulière mise en scène, tirée d'un texte anonyme publié, si l'on en croit la mention d'édition, à Barbe-en-con, en Foutromanie, en l'An des Fouteurs 5796 ? A première vue, la pièce s'inscrit, d'une part, dans la mouvance des tragédies burlesques et érotiques dont les scènes clandestines du Paris du XVIII ${ }^{\mathrm{e}}$ siècle se régalaient, et d'autre part, surtout, dans la vogue des pamphlets révolutionnaires qui pullulèrent durant cette période ${ }^{4}$. Cependant, l'appareil de notes qui accompagne la tragédie, plus volumineux que la pièce elle-même, a de quoi surprendre davantage. En effet, en marge de l'aspect éminemment libre et licencieux de ce texte contre-révolutionnaire, s'élève un monument d'érudition, formé de 175 notes historiques et rapprochements divers, le tout rédigé dans un style extrêmement sérieux où se nouent d'étonnants parallèles entre ces scènes de débauche et l'histoire de la France, entre l'actualité politique et des figures allégoriques dont les attributs dévoyés s'inscrivent dans une tradition séculaire.

Dans un contexte où la France, la Vendée et l'Angleterre sont autant de figures qui s'incarnent sur une scène érotique pour mieux faire ensuite l'objet d'une glose savante, toute la pièce se joue sur deux niveaux de lecture : d'une part, la lecture littérale, qui s'intéresse aux dialogues licencieux, et de l'autre, l'apparat critique, qui dévoile le sens prétendument caché des scènes. Ce dispositif herméneutique requiert évidemment que l'on s'interroge sur le caractère allégorique de la France foutue, où se côtoient la personnification et la métaphore continuée au profit d'une œuvre paraissant à une époque qui, la critique contemporaine l'a souvent rappelé, est précisément marquée par le déclin de l'allégorie, favorisé pendant tout le siècle par un certain désenchantement du monde ${ }^{5}$. Comment expliquer le recours à l'allégorie et, de

3 Ibid., Acte III, sc. 1, p. 76.

4 Le registre de la licence et de l'érotisme semble en effet avoir inspiré bon nombre de pamphlets révolutionnaires. L'on peut signaler, à titre d'exemples, Les Amours de Charlot et Toinette (ca. 1789), Les travaux d'Hercules, ou la Rocambole de la fouterie (ca. 1790), Les fureurs utérines de Marie-Antoinette, femme de Louis XVI (ca. 1791), Les fouteries chantantes, ou les récréations priapiques des aristocrates en vie (vit) (ca. 1791) et L'Écho foutromane (ca. 1792).

5 Au sujet de l'allégorie dans le théâtre français des Lumières, voir ces deux articles de 
surcroît, à l'allégorie obscène, alors qu'il s'agit de penser et de dramatiser l'actualité révolutionnaire? Dans pareil contexte, il apparaît éclairant de montrer de quelle façon la tradition allégorique, qui se prolonge sous une figure dévergondée dans La France foutue, vient alimenter une compréhension de l'histoire, elle-même doublée dans son efficace par l'immense apparat critique qui non seulement permet de rendre explicite l'allégorie, mais donne également à voir un commentaire historique documenté à la faveur d'un exercice rhétorique canonique : le parallèle oratoire.

Le XVIII ${ }^{e}$ siècle a assisté, la critique actuelle l'a bien montré, à un déplacement du genre allégorique. En effet, cette figure qui, chez Quintilien, désignait une "métaphore prolongée ${ }^{6}$ » évolue dès le XVII ${ }^{e}$ siècle vers la sphère profane où elle se déploie dans une rhétorique mondaine du tour ingénieux, elle-même caractérisée par un goût pour les devises, énigmes et autres jeux de mots ; bref, autant d'ornements au service d'une mondanité qui relègue à l'arrière-plan les vastes ambitions heuristiques et théologiques de la tradition exégétique qui inspiraient encore les usages de l'allégorie à la Renaissance. Profondément tributaire de l'essor de la science moderne et du triomphe du rationalisme, le désenchantement de l'allégorie s'accompagne fréquemment, au XVIII siècle, d'une critique hyperbolique qui lui confère, au même titre qu'aux mythes et aux canons classiques, un statut profane et ludique. Indissociable de l'ingenium, c'est-à-dire d'un art mondain de l'allusion critique ou du rapprochement séducteur, l'allégorie devient de plus en plus, suivant l'expression de Sylvain Menant, une «forme spirituelle de la lucidité ${ }^{7}$. Ce processus de désenchantement semble conduire, ultimement, à une subversion dont $L a$ France foutue offre un exemple des plus frappants. Toutefois, la personnification allégorique à l'œuvre dans ce pamphlet royaliste va au-delà d'une simple figure ornementale : l'allégorie n'est pas obscure ; elle est encore moins ingénieuse. Cette mise en scène des troubles révolutionnaires qui se manifeste à la faveur $d^{\prime}$ 'une

Nathalie Rizzoni, "Quand l'Absence apparaît... L'allégorie au théâtre au dixhuitième siècle», SVEC, 7, 2003, p. 429-443 ; «La Parodie en personne : enjeux et jeux d'une figure allégorique au théâtre», dans Dominique Quéro et Sylvain Menant (dir.), Séries parodies au siècle des Lumières, Paris, Presses de l'Université Paris-Sorbonne, coll. «Lettres françaises», 2005, p. 71-86.

6 Quintilien, Institution oratoire, texte établi et traduit par J. Cousin, Paris, Les Belles Lettres, "Collection des universités de Paris», 1975, Livre IX, II.

7 Sylvain Menant, «La raison et l'allégorie au XVIII siècle», dans L'Allégorie, Paris, PUF, coll. «Corps écrit», 1986, p. 95. 
histoire de bordel s'écarte considérablement, d'une part - et de toute évidence - de l'ancien vocabulaire de l'exégèse biblique et, de l'autre, du caractère mondain que l'on attribue habituellement au genre allégorique à l'époque des Lumières. Tout indique que l'allégorie, dans cette tragédie, est un choix esthétique permettant de dramatiser - et par conséquent de comprendre - un événement contemporain, en l'occurrence la trahison du duc d'Orléans secondé par l'Angleterre. Dès lors qu'elle permet de se figurer un rapport au monde, il semble bien que la forme allégorique ait repris, dans ce contexte, l'une de ses anciennes fonctions : celle de donner à lire une vérité voilée, et qu'en même temps l'on ait subverti son usage au point d'en faire une allégorie «foutative», mais néanmoins historique. Pour s'en persuader, il suffit d'observer de quelle façon le genre allégorique vient féconder un imaginaire nourri par une volonté de saisir, d'un point de vue contre-révolutionnaire, les troubles des années 1790 .

L'interprétation des événements révolutionnaires apparait, dans $L a$ France foutue, à la lumière de deux tableaux superposés, à savoir la scène érotico-clandestine et la scène parodique. De fait, le dévergondage de la tradition allégorique dans cette tragédie n'est pas sans rappeler le destin tout aussi burlesque qu'a connu, par exemple, le canon de la tragédie classique sur la scène clandestine française du XVIII ${ }^{e}$ siècle. Ainsi, la France foutue met-elle en scène un très original enchevêtrement entre allégorie politique - ou historique - et allégorie érotique ou - encore foutative - qui vient alimenter le commentaire sur l'histoire. A cet égard, la définition d'une république que formule l'Angleterre, cette maquerelle qui vient «bordéliser la France ${ }^{8}$, est fort éclairante :

Seigneur, j'ai cru devoir vous donner trois rivaux :

Vous savez qu'au bordel les fouteurs sont égaux.

L'on y confond les rangs ; et pour vous mettre à l'aise,

Le premier qui fourbit est le moins bande-à-l'aise.

$\mathrm{Qu}$ 'il soit sujet ou roi, grand, petit, riche ou non,

Quand son nerveux priape a fait bander un con,

Princes des autres vits il a la préférence 9 .

8 La France foutue, op. cit., acte I, sc. 1, p. 32.

9 Ibid., acte I, sc. 5, p. 44 . 
L'allégorie de la république, ce bordel où tous les fouteurs sont égaux, vient ici servir une interprétation des événements contemporains.

D'un autre côté, l'appareil de notes permet souvent de rendre claire l'allégorie. C'est le cas, notamment, dans cet extrait pour le moins insolite où l'Amérique, entre autres, prend les traits d'une femme que l'on entretient ${ }^{10}$ :

Grace à mon greluchon j'ai fixé ma fortune ${ }^{11}$ :

Je ne crains pas du sort la rigueur importune ;

Il régit mon bordel, et lui seul, nuit et jour, S'occupe du tribut de mes filles d'amour ${ }^{12}$.

Et l'Écosse et l'Irlande, et d'autres concubines ${ }^{13}$,

$\mathrm{Qu}^{\prime} \mathrm{il}$ fait foutre à son gré par de lubriques pines ${ }^{14}$,

Mettent en mon pouvoir l'or de leurs coups de culs,

Fruit salutaire et sûr de ses heureux calculs.

La féconde Amérique, et l'Inde fastueuse,

Qu'à force de branler il rendit amoureuse ${ }^{15}$,

Sont aussi des putains que je fais exploiter ${ }^{16}$ :

Ainsi dans mon bordel je puis donc les compter.

10 Nous reproduirons ici les notes historiques qui accompagnent parfois certains vers et qui apparaissent en annexe de la tragédie.

11 «Graceà mon greluchon... Monsieur Pitt, premier ministre du roi d'Angleterre. Il disait un jour devant des ambassadeurs : Nous voulons être les maitres de la mer, et non pas les tyrans. Ce sont les Carthaginois de notre siècle, qui, s'étant rendus maîtres de la mer, défendirent d'y voyager. Un greluchon est un salarié d'un bordel ou d'une catin. Le nom de bordel dérive de bord, qui est un mot saxon ; ce fut en Saxe où l'on connut les premiers bordels». Ibid., p. 100-101.

12 «S'occupe du tribut de mes filles d'amours... Schires, ou comtés de l'Angleterre. Il sont au nombre de cinquante-deux. Londres est dans le comté de Middlesex». Idem.

13 «Et l'Écosse et l'Irlande... Ce sont deux royaumes réunis à l'Angleterre. Et d'autres concubines... Isles circonvoisines de l'Angleterre, et celles de l'Amérique et de l'Inde». Idem.

14 "Qu'il fait foutre à son gré par de lubriques pines... Allusion aux gouverneurs". Idem.

15 "Qu'à force de branler il rendit amoureuse... Monsieur Pitt, de son cabinet, régit les îles de l'Amérique et même commande dans les Indes. L'on peut assurer que c'est lui qui tient l'équilibre de l'Europe. Aussi l'anglais est-il plus fier que jamais : parce qu'il est guerrier, commerçant, philosophe, il croit voir Rome, Carthage et Athènes dans Londres». Idem.

16 «Sont aussi des putains que je fais exploiter... Allusion à l'exploitation des mines d'Amérique». Ibid., p. 102. 
Leurs cons propres et beaux sont des trésors immenses,

Et qui pourraient eux seuls suffire à mes dépenses ;

Leur motte est rebondie, et le poil en est blond ${ }^{17}$ :

Il faudrait un fier vit pour en trouver le fond $!^{18}$

Cette longue métaphore filée sur les richesses du royaume d'Angleterre et de l'Amérique, en particulier, se déploie au sein d'une allégorie foutative où les royaumes régis par la maquerelle s'incarnent en putains. De cette façon, le recours à l'allégorie, indissociable d'un chassé-croisé entre le texte et l'appareil de notes, permet de penser l'histoire à travers d'étonnantes figures de la lubricité.

La personnification allégorique de la Vendée, région qui s'est battue corps et âme contre l'armée républicaine, semble aussi donner lieu à une interprétation de l'expérience contemporaine. Voici un dialogue entre d'Orléans et l'Angleterre :

L'Angleterre $^{19}$ :

Avez-vous vu Puisaye?

D'Orléans :

Auprès de la Vendée ${ }^{20}$

Je l'ai laissé, Madame. Elle reste attachée

A sa jeune maîtresse, et rien encore n'a pu

Lui faire décider à lui tourner le cu.

L'Angleterre :

Je connais cette fille ; elle est jeune, elle est belle,

Et comme sa maîtresse, elle est encor pucelle ${ }^{21}$

Par cet enlèvement les forçant toutes deux,

Le comte seul l'aura, $\mathrm{s}^{\prime}$ il en est amoureux ${ }^{22}$.

Je dois la protéger, et notre politique

17 «Leur motte est rebondie, et le poil en est blond... Allusion aux montagnes de l'Amérique et à la couleur de l'or». Ibid., p. 102.

18 Ibid., acte I, sc. 1, p. 26-27.

19 Ibid., acte II, sc. 2.

20 «Auprès de la Vendée... Allusion à cette partie de la France que j'ai personnifiée». Ibid., p. 125.

21 «Et comme sa maitresse, elle est encor pucelle... Royale et loyale». Idem.

22 «Le comte seul l'aura, s'il en est amoureux... Le comte de Puisaye a commandé dans l'armée catholique». Idem. 
Est d'en faire à son tour une fille publique ${ }^{23}$.

Je connais ses besoins, et voulant y pourvoir, Je saurai la gagner par quelque faux espoir.

Alimentant son cœur assez sans passer outre, Lorsqu'il en sera tems je la lui ferai foutre ${ }^{24}$.

«Lorsqu'il en sera tems je la lui ferai foutre» : grâce à une rhétorique du double sens, l'auteur passe ici du commentaire allégorique très érotique - à travers la mise en scène du viol prochain de la Vendée personnifiée - au commentaire historique et politique, c'est-à-dire à la défaite de la Vendée et des chouans.

A l'évidence, l'interprétation de l'histoire se joue sur deux niveaux : alors que la parodie de la pratique allégorique permet de présenter les événements révolutionnaires sous les traits d'une anecdote licencieuse, la glose savante donne lieu, pour sa part, à des réflexions dont l'érudition, qui s'inscrit dans la longue tradition rhétorique du parallèle oratoire, se pose comme garant d'une compréhension de l'histoire. Pour un royaliste, le royaume de France a été trompé - ou foutu ; ainsi, la personnification allégorique d'une France foutue représente-t-elle au mieux les événements révolutionnaires et permet de se les figurer. Alors que la période révolutionnaire - comme, par ailleurs, d'autres épisodes critiques de l'histoire de France - voit exploser un genre comme celui du dialogue des morts, écrit sous forme de parallèles entre morts anciens et modernes, il apparaît clairement que les notes historiques permettent de décrypter l'allégorie, mais aussi et surtout que les rapprochements entre divers événements qu'esquisse l'auteur à la fin de sa tragédie viennent renforcer cette ambition de comprendre les troubles qui ont conduit à la naissance de la République.

En annexe de la pièce, les «rapprochements de circonstances» consistent en de courts paragraphes où la prémisse du raisonnement - plus souvent qu'autrement puisée dans le vaste fond antique - est suivie d'un commentaire sur l'actualité. Alors qu'il n'est nullement question, dans la tragédie, de la présence de Bonaparte (les événements retracés

23 «Est d'en faire à son tour une fille publique... Il se rendit en Vendée des hommes de tous les partis, ce qui accéléra sa perte». Idem. Cependant, la «fille publique» peut aussi faire écho à la République.

24 «Lorsqu'il en sera temps, je la lui ferai foutre... L'Angleterre paya le comte de Puisaye, et s'en servit pour perdre la Vendée et les chouans. Une des premières familles de la Bretagne a prétendu que le comte de Puisaye avait fait fusiller et guillotiner une partie de la noblesse du pays, par les bleux». Idem. 
dans la pièce touchent principalement à l'échec de la Vendée grâce à la conspiration de d'Orléans secondé par l'Angleterre), les rapprochements semblent avoir été, pour leur part, écrits plus tard et, selon toute vraisemblance, au début du consulat de Napoléon $1^{\text {er }}$ en 1802 . En voici quelques extraits choisis, qui mettent bien en lumière la fonction du parallèle oratoire dans un processus heuristique de compréhension de l'histoire :

Prémisse : «L'Empereur Nerva n'était ni romain, ni originaired'Italie, il n'était pas moins empereur de Rome».

Commentaire : «Pourquoi les Français se plaindraient-ils d'être gouvernés par un étranger ? est-il plus coupable que ceux qui ont assassiné leur roi légitime? son usurpation ne légitime pas plus leurs crimes, que leurs crimes légitiment son usurpation ${ }^{25} »$.

Prémisse : «Sénèque fut exilé en Corse, par Néron».

Commentaire : «Pourquoi le premier consul ne s'est-il pas fait faire une généalogie semblable : il est encore en France des généalogistes, et le peuple, ami du merveilleux, y eût plutôt cru qu'à celle de Milan. Ce n'est point impunément qu'on en impose à son siècle ${ }^{26}{ }^{26}$.

Prémisse : «Sylla fit égorger les six mille prisonniers samnytes, dans le cirque à Rome, pendant qu'il haranguait le peuple au temple de Bellone».

Commentaire : «Tandis qu'on pacifiait les chouans à Paris, 1 'on fusillait le brave général Frotté et son état-major ${ }^{27}{ }{ }$.

Ces trois parallèles historiques illustrent au mieux la pratique d'une figure oratoire qui permettait, pendant tout l'âge classique, de se penser «au sein d'un dialogue ininterrompu avec les Anciens ${ }^{28}$ ». De fait, "cette figure singulière tire son crédit et son efficace $d^{\prime}$ 'une promesse : celle de rendre intelligible l'histoire moderne de l'Europe en lui conférant la forme d'un 'argument' se soutenant d'un rapprochement, par-delà les

25 Ibid., p. 169.

26 Idem.

27 Ibid., p. 174.

28 Marc André Bernier, «Entre progrès et inquiétude : les figures de la fin de la civilisation au siècle des Lumières" ; communication présentée à l'occasion du colloque tenu dans le cadre du $66^{\mathrm{e}}$ Congrès de $\mathrm{l}^{\prime}$ ACFAS sous le thème "Les imaginaires de la fin»; Québec, Université Laval, 12 mai 1998, p. 3. 
siècles, avec $l^{\prime}$ histoire antique ${ }^{29}{ }{ }$. Ce faisant, le parallèle oratoire se déploie à la faveur d'une «réversibilité» qui permet de "penser aussi bien le temps historique que le politique ${ }^{30}$ » et dont le parallèle entre Nerva et Napoléon, dans le premier rapprochement, constitue un exemple éloquent.

Placés sous ce jour, la chute de la monarchie française et les débuts de la République semblent faire écho à la fin de l'ancien Empire romain ; la France monarchique déchue peut donc relire sa propre disgrâce à la lumière $d^{\prime}$ 'un souvenir historique, comme en témoigne ce très bel extrait de la longue tirade prononcée par la France dès lors qu'elle perd sans retour sa virginité, symbole d'une souveraineté inaliénable :

Le français, qui jadis du monde était l'exemple !

Le français révolté que l'Europe contemple,

Après un tems si long de quatorze cents ans,

Ose se comparer à d'illustres brigands [les Romains] !

A ce peuple assassin, qui, révoltant la terre,

S'entr'égorgeait lui-même, en se traitant de frère :

Où la nature avait perdu ses droits si beaux,

Où cette liberté dressa des échafauds :

Où de Coriolan la vertu fut punie,

Où l'un des décemvirs condamna Virginie :

Où le père envoya son enfant à la mort :

Où César de son fil reçut le même sort :

Où la proscription fit nombre de victimes,

Et qui, de crime en crime, inventa tous les crimes.

Voilà donc le modèle où le français prétend :

Il veut être romain !...ah ! qu'il est inconstant ${ }^{31}$ !

De vierge qu'elle était, la France est devenue république ou "garce publique», violée au sein de son propre royaume, rappelant dès lors la Rome antique, comme le rappelle ce fragment d'un dialogue entre d'Orléans et l'Angleterre où la stichomythie, procédé dramatique qui,

29 Marc André Bernier, "Quand le présent prend la figure des Anciens», dans Thierry Belleguic (sous la dir. de), Fables du temps au siècle des Lumières, Québec, Presses de l'Université Laval, coll. «République des Lettres», sous presse, p. 6.

30 M. A. Bernier, «Entre progrès et inquiétude : les figures de la fin de la civilisation au siècle des Lumières", loc. cit., p. 6.

31 La France foutue, op. cit., acte III, sc. 1, p. 76-77. 
par ailleurs, met en scène, dans la tragédie classique, le choc de deux orgueils, se trouve parodiée dans le récit de ce complot, dont le caractère au demeurant fort tragique n'est pas sans rappeler les parodies érotiques clandestines du théâtre de Corneille ${ }^{32}$ :

D'Orléans :

Que faut-il faire?

L'Angleterre :

Il faut...

D’Orléans :

Quoi ?...

L'Angleterre :

La foutre aujourd'hui.

D'Orléans :

Comment? et dans quel lieu ? quand ?

L'Angleterre :

Quand? dans quel lieu, Prince?

Vous me parlez, hélas! en fouteur de province.

Rome le fut dans Rome, et la France en ce jour,

Doit l'être dans Paris, au milieu de sa cour ${ }^{33}$.

De même, l'Angleterre ne dit-elle pas ouvertement au sujet de la France, au dernier acte, qu'elle «en [veut] faire à son tour une autre Messaline»? Autant de parallèles entre l'histoire ancienne et les événements contemporains soulignent bien l'importance, pendant tout l'âge classique, d'aller puiser dans le passé de grandes réminiscences capables de mettre en relief la dimension tragique du présent.

Dévergondée, l'allégorie rend donc possible une réflexion sur le présent et contribue, du même coup, à donner un sens à l'histoire contemporaine ; parodiée - ou foutative - elle propose un extravagant dialogue entre politique et érotisme. Ni complètement pamphlétaire, ni tout à fait licencieuse, La France foutue offre un exemple convaincant de la radicalisation d'une pratique rhétorique où personnification allégorique et politique s'entremêlent au service d'une lecture de l'événement historique. D'ailleurs, l'auteur n'affirme-t-il pas lui-même, à la fin de sa pièce : «J'ai écrit mon poëme avec une plume libidine, et $j$ 'ai fait une

32 Parodies érotiques telles que La Nouvelle Messaline (ca. 1753) et La Comtesse d'Olonne (ca. 1738) de Grandval fils.

33 Ibid., acte I, sc. 1, p.31. 
priapée, d'événements tragiques [...] j'ai écrit pour les libertins, parce que ce sont ceux-là que je veux qui me lisent. Lorsqu'il s'agit du bien, qu'importe comment on l'opère ? N'avez-vous jamais pris du poison pour vous guérir ? ${ }^{34}$ ».

\section{STÉPHANIE MASSÉ}

Université du Québec à Trois-Rivières

34 Ibid., «Un Mot sur mon Poëme», p. 181. 